\title{
ON THE STRUCTURE AND KINEMATICS OF MOLECULAR CLOUDS FROM LARGE SCALE MAPPING OF MM-LINES
}

\author{
J. Bally, W.D. Langer, R.W. Wilson, A.A. Stark, and M.W. Pound \\ AT\&T Bell Laboratories \\ HOH-L245, Holmdel, NJ 07733
}

\begin{abstract}
Molecular gas in the interior of the Orion superbubble consists of shects, filaments, and partial shells in which the active star forming dense cloud cores are embedded. The main body of the Orion A and B clouds and at least 14 smaller clouds in Orion region are cometary in appearance suggesting strong interaction with massive stars in the Orion $O B$ association. While the small scale $(<1 \mathrm{pc})$ structure of the clouds may be determined primarily by internal magnetic fields, gravity, and the effects of outflows from young stellar objects, the large scale morphology and kinematics is affected by the energy injected by massive stars. Supernovae, stellar winds, and radiation have compressed, accelerated, ablated, and dispersed molecular gas over the last $10^{7}$ years. Most GMC/OB star complexes in the Solar neighborhood exhibit morphological and kinematic properties similar to the Orion region. We argue that energy injection by massive stars plays a vital role in the evolution of the ISM and may be responsible for much of the observed large-scale structure and kinematics of molecular clouds.
\end{abstract}

\section{INTRODUCTION}

Over the last 6 years, the Bell Laboratories 7-meter antenna has been used to observe over 700,000 individual positions in 24 regions of the sky containing molecular clouds. The purpose of this survey is to search for morphological and kinematic clues to the evolution and life cycle of molecular clouds. Large scale mapping of the $\mathrm{J}=1-0$ lines of ${ }^{12} \mathrm{CO}$ and ${ }^{13} \mathrm{CO}$, and to a more limited extent the $\mathrm{J}=2-1$ line of $\mathrm{CS}$, allow investigation of the structure and kinematics of molecular gas on the scale of an entire Giant Molecular Cloud (GMC). In this paper, we analyze the Orion region (based on about 300,000 spectra obtained on a $1^{\prime}$ grid with a 100 " dianeter beam) and apply the results to a discussion of the evolution and star formation history of clouds throughout the Galaxy.

We describe the physical and velocity structure of the Orion region and relate this structure to available sources of energy, exploring the possibility that kinetic energy injection from recently formed stars, combined with the influence of gravity and magnctic ficlds, determines the present state of the Orion clouds. In the last section, we discuss application of these considerations to models of the origins and evolution of molecular clouds on a galactic scale.

\section{MOLECULAR GAS IN THE ORION REGION}

Figure 1 is a color image of the ${ }^{12} \mathrm{CO},{ }^{13} \mathrm{CO}$, and $\mathrm{CS}$ emission from the major concentrations of molecular gas in the Orion region. The images show that most of the $10^{5} \mathrm{M}_{\odot}$ of molecular gas is confined to three major clouds: the Orion A and L1641 cloud in the south, the Crion B cloud containing NGC 2024 and NGC 2023 in the middle, and the NGC 2071 cloud containing NGC 2068 in the north. The large-scale morphology and kinematics of the gas is similar in both ${ }^{12} \mathrm{CO}$ and ${ }^{13} \mathrm{CO}$. However, the filamentary internal structure of the cloud is easier to see in the more optically thin species. Most of the structure in the CO images shows the gas to consist of filaments or sheets and partial shells, suggesting that the gas is responding to 
hydrodynamic forces or magnetic fields.

Fragmentation is evident in $\mathrm{CO}$ or ${ }^{13} \mathrm{CO}$ only on the largest scales, where the gas in the Orion complex can be subdivided into the 3 major clouds and several dozen smaller clouds or cores. In the L1641 region of Orion, only about $5 \%$ of the ${ }^{13} \mathrm{CO}$ emission can be assigned to well defined clumps (Bally et al. 1987). The intensity of ${ }^{13} \mathrm{CO}$ emission ranges from about 3 to $60 \mathrm{~K}$ $\mathrm{km} \mathrm{s}^{-1}$, indicating that the column density of molecular gas towards the major clouds in Orion is about 3 to 5 times greater than towards the Taurus dark clouds (Ungerechts and Thaddeus 1987; Myers and Benson 1983). The higher gas column density and line opacity in Orion renders cloud cores more difficult to see in ${ }^{12} \mathrm{CO}$ or ${ }^{13} \mathrm{CO}$ compared to lower column density cloud complexes such as Taurus or the Rosette Molecular Cloud (Blitz and Stark 1986).

Many individual cloud cores closely associated with star formation can, however, be seen in the high density tracer CS (Lada 1989; Lada, Bally, and Stark 1990). Most clouds exhibit highly filamentary structure in the $\mathrm{CO}$ lines. Cloud cores and fragmentation are best observed in the CS lines or other high-dipole-moment molecules which probe clumping on a scale closer to that on which star formation occurs.

On the smallest scales resolved by our survey $(0.2$ to $3 \mathrm{pc}$ ), we see many small cavities, bubbles, and regions having large ${ }^{13} \mathrm{CO}$ linewidths which may have been produced by molecular outflows or stellar winds from young stellar objects. Examples of these structures include small cavities surrounding the HH 1 and 2 region near NGC 1999, the broad lines seen $20^{\prime}$ north of this region (near the cluster L1641N), and the nearly complete bubble located about $1^{\circ}$ to the southwest of NGC 2068.

On linear scales of a few to tens of parsecs, the cloud consists of a network of filaments and partial shells which have a variety of characteristics. Some of the filaments extend for many degrees but are only a few arc minutes wide - aspect ratios (length / width) in excess of 30:1 are common. Examples of long straight filaments are the ones marking the western edge of L1641, and the southern boundary of the Orion B cloud. The images in Bally et al. (1987) show over a dozen filaments in the Orion A (L1641) cloud which have velocity jumps and reversals along their length, yet remain spatially continuous. The straight filaments may contain magnetic field lines along which gas collects to form "magnetized ropes". Magnetic turbulence in the form of Alfven waves may prevent (or regulate) gravitational collapse along the field lines while magnetic pressure resists flow orthogonal the field lines.

Curved filaments or partial shells are seen in other parts of the cloud. Examples include several overlapping arcs or filaments located $1^{\circ}$ east of NGC 2024, the $1^{\circ}$-long tongue of gas projecting towards the east from between the NGC 2023 and NGC 2024 cloud cores, the "bubble" projecting to the southeast of NGC 2068, several nearly concentric partial shells about $20^{\prime}$ in radius surrounding the NGC 1999 reflection nebula, and "arches" of gas lying about $20^{\prime}$ north of NGC 2071. These features may be limb-brightened partial shells or sheets seen edge-on and may indicate the presence of shocks. Energy release from massive stars or groups drive shock waves into the molecular cloud, altering its structure. These structures suggest that magnetic fields and large scale shocks waves play an important role in determining cloud structure, dynamics, and evolution in the Orion region.

The Orion molecular clouds show organization on the scale of a hundred parsecs. The cloud shapes and velocity gradients have a preferred orientation pointing toward the center of the Orion $\mathrm{OB}$ association, evidence that energy release from these massive stars must have played an important role in the evolution of these clouds. 


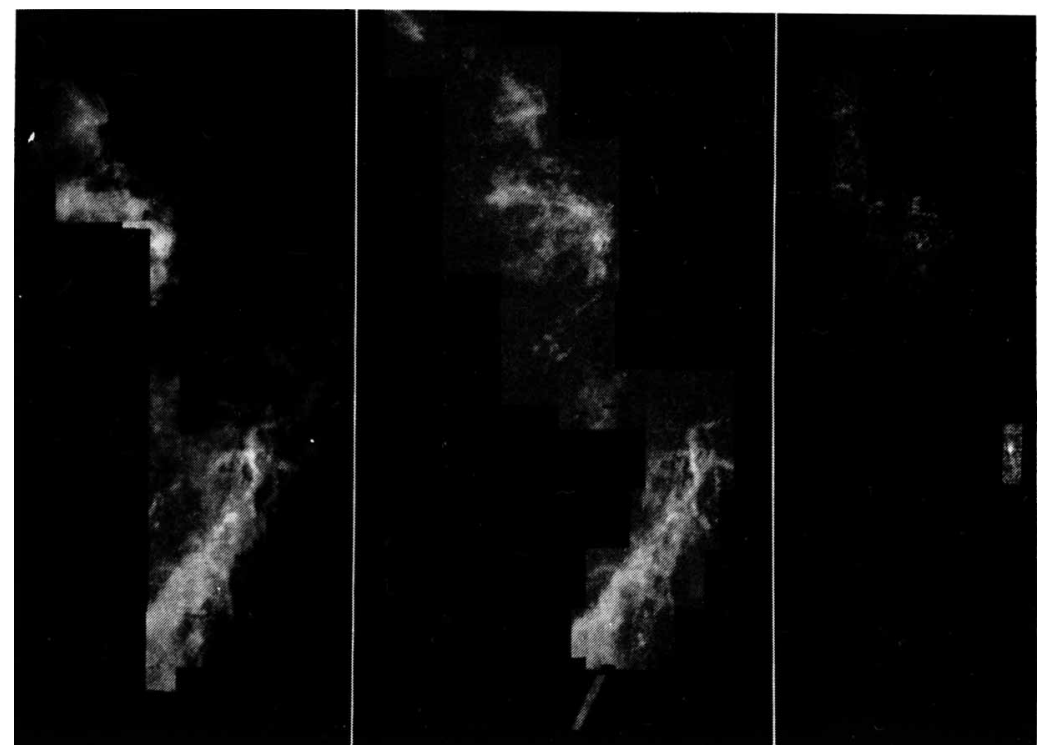

Figure 1: Three color images of the Orion region showing the major molecular clouds in ${ }^{12} \mathrm{CO}$ (left), ${ }^{13} \mathrm{CO}$ (middle), and $\mathrm{CS} \mathrm{J}=2-1$ (right). Each image shows the emission integrated from $V_{\mathrm{LSR}}=-4 \mathrm{~km} \mathrm{~s}^{-1}$ to $\mathrm{V}_{\mathrm{LSR}}=16 \mathrm{~km} \mathrm{~s}^{-1}$, color coded with velocity so that blue corresponds to the velocity interval $(-4,5)$, green $(5,9)$ and red $(9,16)$. The reflection nebulae NGC 2071 and NGC 2068 are located in the main concentration near the top of the figure, NGC 2023 and NGC 2024 is in the center, and Orion A (M42) is the region near the top of the comet-shaped L1641 region at the bottom. (See Color-plates section)

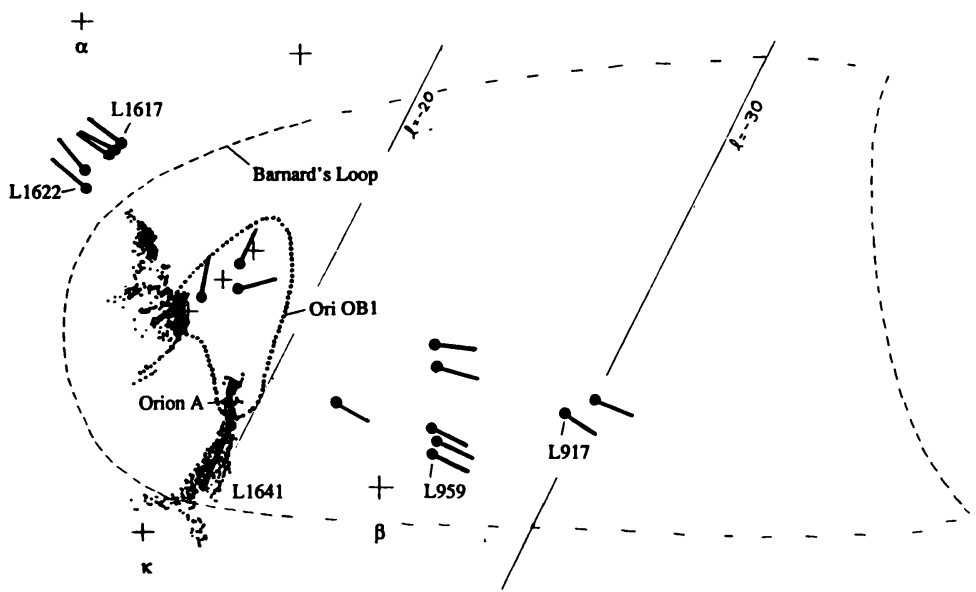

Figure 2: A large-scale view of the Orion region showing the location of Barnard's Loop on the left and the Eridanus Loop on the right which mark the optical edge of the sphere of influence of the Orion OB association. A fragmented shell of $21 \mathrm{~cm} \mathrm{HI}$ emission lies outside the dashed line which marks the position of faint $\mathrm{H} \alpha$ nebulosity. The location of the Orion OB 1 association stars is indicated by the dotted region. The comet-shaped objects mark the location and orientation of 16 cometary molecular clouds. All of these clouds point toward the OB stars located near the center. 


\section{CLOUD SYSTEMATICS IN ORION}

The extent of the sphere of influence of the Orion $O B$ association can be estimated from the size and location of the bubble of gas marked on the east by Barnard's Loop and by the Eridanus Loop $30^{\circ}$ to the west (Sivan 1974). A $21 \mathrm{~cm}$ HI bubble surrounds this region, indicating that at least a $100 \mathrm{pc} \times 300 \mathrm{pc}$ region containing all of the molecular gas discussed above has been influenced by the energy released by the OB stars in the Orion region. Ultraviolet observations of the near side of this expanding shell (Cowie, Songaila, and York 1979) show a typical expansion velocity of order 30 to $50 \mathrm{~km} \mathrm{~s}^{-1}$ in the low density gas seen along the line-ofsight towards the Orion $\mathrm{OB}$ stars.

At least 16 molecular clouds within the boundaries of the Orion superbubble exhibit cometary morphology. These clouds have dense heads with tails pointing away from the center of the Orion $\mathrm{OB}$ association as shown in Figure 2. Even the large clouds in Orion exhibit edges and filaments pointing radially away from the OB stars. These structures can be recognized in both the CO and IRAS infrared images of the Orion region. As shown by Bally et al. (1987), the entire southern part of the Orion A cloud (L1641) has a cometary morphology.

Figure 3 shows the detailed morphology of eight cometary clouds in the Orion region. Five clouds which lie in the northeastern portion of Barnard's Loop are shown in the two panels on the left. These clouds point towards the southwest, towards the center of the OB association. The cometary cloud L1622 is surrounded by a "boomerang"-shaped ridge of strong $12 \mu \mathrm{m}$ emission which coincides with the optically bright southwestern edge of the cometary cloud. This feature indicates that strong radiation fields illuminate the cloud from the direction of the Orion $\mathrm{OB} 1$ association, which is in the opposite direction from the Galactic plane. A small cluster of low luminosity stars has formed in the head of this cometary cloud. The overall shape, kinematics, and morphology resembles the models of cometary clouds produced by ionizing radiation discussed by Bertoldi (1989a,b) and Bertoldi and McKee (1990).

These clouds are located slightly outside Barnard's Loop, which marks the present location of the ionization front generated by the OB association in the low density gas located between the major molecular clouds. Since the gas near L1622 and L1617 has already been extensively altered by interaction with the OB stars, it seems likely that in the past, radiation from an $\mathrm{OB}$ association has extended beyond the present location of Barnard's Loop. As new OB stars are born and die, the Lyman continuum luminosity of the association fluctuates, producing ionization fronts which repeatedly expand and retreat. Also, the cometary globules in this portion of the Loop may have originated well inside Barnard's Loop and been accelerated radially outward by the rocket effect.

The upper and lower right panels of Figure 3 show two cometary clouds in Eridanus which again point toward the Orion OB association. L917 and L906 are located nearly $30^{\circ}$ below the plane, which at the distance of Orion corresponds to about $300 \mathrm{pc}$ or over 4 molecular cloud scale heights from the galactic plane. These clouds must have been either ejected from near the main Orion clouds or condensed from the expanding HI shell surrounding Orion.

There are several systematic patterns in the morphology and kinematics of the gas in Orion: (1) Large scale velocity gradients point towards the center of the $\mathrm{OB}$ association. In the Orion A, Orion B, and in the NGC 2071 clouds, the portion of the cloud closest to the Orion OB association (whose oldest sub-group is centered about 3 degrees west of NGC 2024) have the most positive velocity (about $\mathrm{V}_{\mathrm{lsr}} \approx 10 \mathrm{~km} \mathrm{~s}^{-1}$ ) while most of the gas farther from the OB stars has a velocity in the range $V_{\text {lsr }} \sim 1$ to $8 \mathrm{~km} \mathrm{~s}^{-1}$. (2) The densest parts of the molecular clouds face the $\mathrm{OB}$ association. Eight degrees south of the $\mathrm{OB}$ association, the southern part of the Orion $\mathrm{A}$ cloud has a width of about $1.5^{\circ}$ and a mean density of about $10^{3} \mathrm{~cm}^{-3}$. Four degrees 

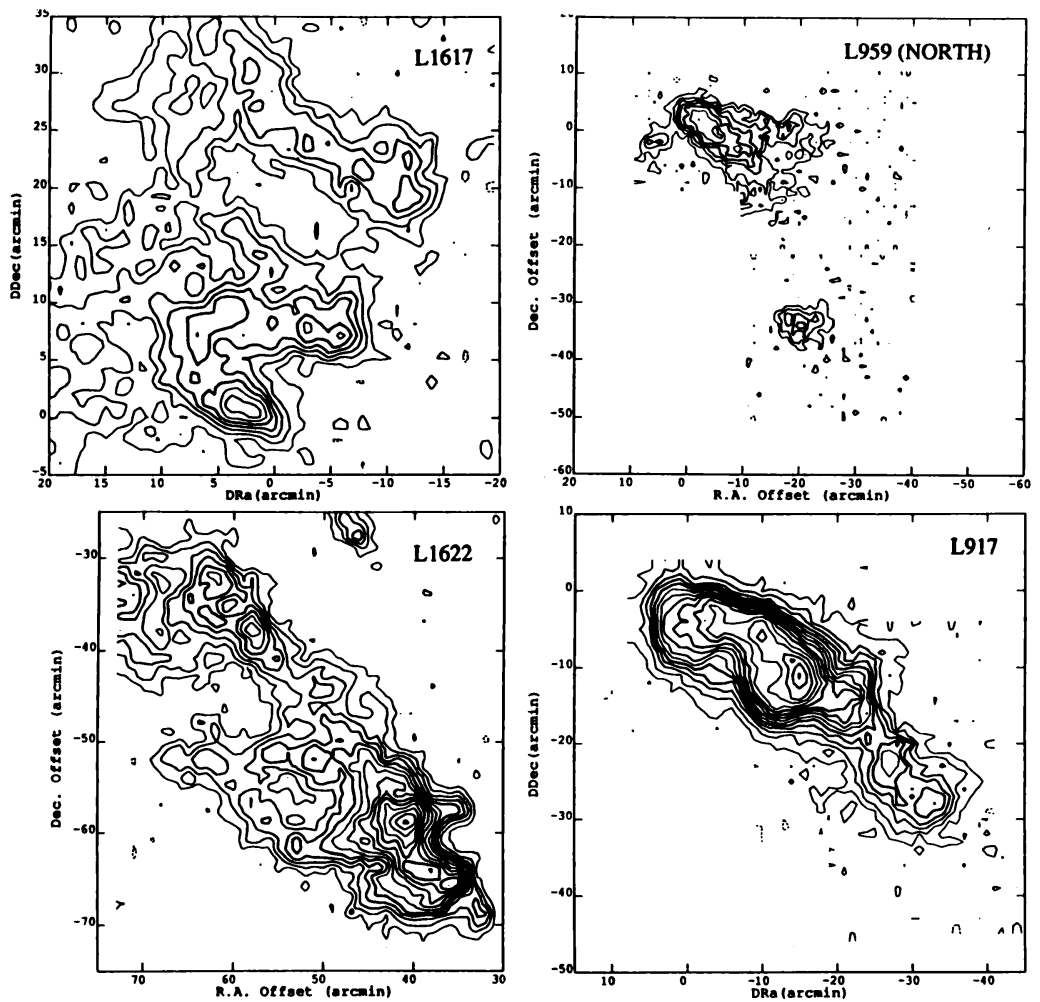

Figure 3: Four panels showing eight different cometary clouds in the Orion region. Note that the objects shown on the left, which lie to the northeast of the Orion $\mathrm{OB}$ association point to the southwest while the objects shown on the right, which lie on the other side of the OB stars point in the opposite direction.

(Upper Left) A group of 3 cometary clouds in Lynds 1617, which contain the Herbig-Haro Objects HH-110, HH-111, and HH-113 (Reipurth 1989). Map shows ${ }^{13} \mathrm{CO}$ integrated from $\mathrm{V}_{\mathrm{LSR}}$ $=8$ to $10 \mathrm{~km} \mathrm{~s}^{-1}$. Contour interval is $1 \mathrm{~K} \mathrm{~km} \mathrm{~s}^{-1}$ and the $(0,0)$ position is $\alpha(1950)=05^{\mathrm{h}} 49^{\mathrm{m}} 09^{\mathrm{s}}$, $\delta(1950)=02^{\circ} 47^{\prime} 50^{\prime \prime}$, the source of the HH-111 jet.

(Lower Left) The cometary cloud L1622. At least 6 low mass stars have formed near the head. A smaller cometary globule is seen near the top of the figure. Map shows ${ }^{13} \mathrm{CO}$ integrated from $V_{\mathrm{LSR}}=0$ to $3 \mathrm{~km} \mathrm{~s}^{-1}$. Contour interval is $1 \mathrm{~K} \mathrm{~km} \mathrm{~s}^{-1}$ and the $(0,0)$ position is the same as in the upper left panel.

(Upper Right) Two cometary clouds located to the west of M42 in L959. Map shows ${ }^{13} \mathrm{CO}$ integrated from $V_{L S R}=-4$ to $0 \mathrm{~km} \mathrm{~s}^{-1}$. Contour interval is $1 \mathrm{~K} \mathrm{~km} \mathrm{~s}^{-1}$ and the $(0,0)$ position is $\alpha(1950)=05^{\mathrm{h}} 05^{\mathrm{m}} 00^{\mathrm{s}}, \delta(1950)=-6^{\circ} 18^{\prime} 00^{\prime \prime}$.

(Lower Right) A large cometary cloud, L917, located 29 degrees below the Galactic plane in Eridanus. Assuming a distance of $500 \mathrm{pc}$, this cloud lies $300 \mathrm{pc}$ outside of the Galactic plane. Map shows ${ }^{12} \mathrm{CO}$ integrated from $\mathrm{V}_{\mathrm{LSR}}=-6$ to $-4 \mathrm{~km} \mathrm{~s}^{-1}$. Contour interval is $1 \mathrm{~K} \mathrm{~km} \mathrm{~s}^{-1}$ and the $(0,0)$ position is $\alpha(1950)=04^{\mathrm{h}} 46^{\mathrm{m}} 20^{\mathrm{s}}, \delta(1950)=-5^{\circ} 52^{\prime} 00^{\prime \prime}$. 
south of the association, near M42, the emission is concentrated into a narrow ( $5^{\prime}$ wide) $\int$-shaped filament which is bright in the $\mathrm{J}=2-1 \mathrm{CS}$ line, implying an average density $\mathrm{n}>10^{4} \mathrm{~cm}^{-3}$. In the Orion $B$ region, the western edge of the cloud contains a CS-bright compressed ridge facing the HII region IC434 (which lies due west of the Horsehead Nebula) while several degrees to the east, farther away from the $\mathrm{OB}$ association, the cloud consists of low-density filaments. Further to the north, the NGC 2071 cloud contains dense cores facing toward the southwestem edge of the cloud. Here too, the average density drops with increasing distance from the OB association. (3) The most active star formation has taken place in the dense gas nearest the OB association. Near infrared surveys conducted with $2 \mu \mathrm{m}$ cameras demonstrate the existence of four dense concentrations of embedded young stars in Orion B. Dense clusters are also known to exist in M42, NGC 1977, and in the northern part of L1641, in the Orion A cloud. These clusters contain between 50 and 1000 individual members with core densities approaching 1000 stars per cubic parsec. (See Lada 1989 for the NGC 2023, NGC 2024, NGC 2068, and NGC 2071 clusters). Inspection of co-added IRAS images at $12 \mu \mathrm{m}$ shows large concentrations of stars throughout most of the dense CS emitting regions of the Orion clouds.

\section{ORIGIN OF KINETIC ENERGY IN MOLECULAR CLOUDS}

The above observations indicate that there may be a connection between the structure, morphology, and kinematics of the Orion clouds and identifiable stellar energy sources. On small scales $(-0.1$ to $1 \mathrm{pc})$, outflows from young stellar objects produce shocks, sweep-up gas into compressed shells, and inject kinetic energy which may contribute to the regulation of cloud collapse. On $\sim 100 \mathrm{pc}$ scales, the Orion clouds are shaped, accelerated, and compressed by the collective effect of shocks and ionizing radiation produced by stars in the OB association. On the largest scales (>100 pc) important effects are: gravitation, tidal shear manifested in the differential rotation of the Galaxy, mutual magnetic and gravitational interaction between giant molecular clouds, spiral density waves, and expanding supershells. The kinetic energy content of clouds is ultimately derived from the cumulative effects of all of these influences.

In active star forming regions like Orion, the combined effects of individual stars on the gas and the cumulative influence of the entire $O B$ association over its lifetime result in the injection of kinetic energy on all scales, ranging from several hundred parsecs to under a parsec. Therefore, the spatial and velocity structure on the small scales can not be viewed as the result of a turbulent cascade which transports energy from the largest scale to the smallest. As can be seen from the cometary clouds located over $15^{\circ}$ away from the center of the OB association, stellar energy injection can operate over large distances. The dependence of the observed linewidth on the size of the region sampled can be described as a power law, $\langle\Delta V\rangle \alpha\left\langle r^{\alpha}\right.$ where $\alpha=0.3 \pm 0.1$. Although this power law often characterizes turbulent cascades, it may be produced by other mechanisms. For example, this size-linewidth relationship naturally occurs in an ensemble of virialized clouds having mean density scaling as a power law of the size, $\langle\rho\rangle \propto\left\langle\mathrm{r}^{2(\alpha-1)}\right.$.

\section{(a) Energy Injection in Orion}

Stars can inject kinetic energy or momentum by several mechanisms: (1) Shock waves generated by embedded outflows from young stellar objects, (2) stellar winds and supernovae produced by older stars located in the vicinity of the cloud, and (3) ionization by hard UV radiation.

On small scales, winds and outflows from young stellar objects can produce 0.01 to 1 pc cavities inside molecular clouds. There are several dozen optical and molecular outflows in the Orion region, injecting momentum into the cloud at a rate 


$$
\dot{\mathrm{P}}_{\text {flow }}=0.05 \mathrm{M}_{\odot} \mathrm{km} \mathrm{s}^{-1} \mathrm{yr}^{-1}\left[\frac{N_{\text {flow }}}{20}\right]\left[\frac{M_{\text {flow }}}{2.5 \mathrm{M}_{\odot}}\right]\left[\frac{V_{\text {flow }}}{10 \mathrm{~km} \mathrm{~s}^{-1}}\right]\left[\frac{10^{4} \text { years }}{\tau_{\text {dyn }}}\right]
$$

where $N_{\text {flow }}$ is the number of flows, $M_{\text {flow }}$ is their average molecular mass, and $V_{\text {flow }}$ is their average characteristic flow velocity. The Orion clouds contain about $10^{5} \mathrm{M}_{0}$ of molecular gas with a typical line FWHM of order $3 \mathrm{~km} \mathrm{~s}^{-1}$. The time required to supply the momentum contained in the gas is $P / \dot{P} \approx 6 \times 10^{6}$ years. Fukui (1989) discuss the role outflows play in regulating cloud collapse.

On large scales, shocks driven by supernovae or main sequence winds of massive stars sweep up low-density intercloud gas lying in front of the shock into a high density snowplowed layer, while the tenuous plasma behind the shock is heated, raising the post shock pressure. The Orion region has produced several dozen $O$ stars which have exploded as supernovae in the last $10^{7}$ years (Blaauw 1964), releasing roughly $10^{51}$ ergs of energy about every $5 \times 10^{5}$ years. During their main sequence phase these stars have already injected a comparable amount of energy in the form of stellar winds. Thus the mechanical luminosity of the Orion $\mathrm{OB}$ association is at least $2 \times 10^{51} \mathrm{ergs} / 5 \times 10^{5}$ years $\approx 3 \times 10^{4} \mathrm{~L}$. Since the $\mathrm{O}$ stars lie close to the molecular clouds from which they were born, the clouds subtend about $10 \%$ of the sky as seen from the star. Thus the kinetic energy coupled into the cloud is $\eta \cdot 3 \times 10^{3} L_{\odot}$, where $\eta$ is the efficiency with which the SN or wind energy is converted to kinetic energy of motion in the cloud. An efficiency of $\eta=0.003$ implies a kinetic energy injection rate of $10 \mathrm{~L}_{\odot}$ into the clouds which would generate the internal turbulent energy of the cloud $\left(\sim 10^{49} \mathrm{ergs}\right)$ in about $8 \mathrm{Myr}$.

Since the supershell shocks propagate rapidly into the low-density ISM and move slowly into denser clouds, these clouds are surrounded and encorporated into the interior of the bubble. Initially, the interior of the bubble is heated to a temperature of about $10^{6} \mathrm{~K}$. Cooling by radiation, electron conduction, and the evaporation of cold clouds within the cavity lowers the internal temperature over an expansion time scale. Since reheating occurs periodically because of the explosion of new supernovae and the steady input of energy from the winds of massive stars, the interior of the large bubbles surrounding $\mathrm{OB}$ associations will have a higher pressure that the surrounding ISM. This effect helps to compress clouds (cloud crushing - Bedogni and Woodward 1990), possibly triggering gravitational collapse and star formation.

Ionizing radiation heats gas to $10^{4} \mathrm{~K}$ in the process of $\mathrm{HII}$ region formation. Most HII regions develop in highly non-uniform environments, which result in the the development of highly anisotropic motions in the ionized medium (the "champagne flow" of Bodenheimer, Tenorio-Tagle, and York 1979) with velocities close to the sound speed in the ionized gas $(\sim 10$ $\mathrm{km} \mathrm{s}^{-1}$ ). As the average density of the HII regions decreases due to expansion, an ever-increasing fraction of the neutral cloud is ionized. A reaction force on the neutral cloud results which can compress and accelerate the neutral cloud to a velocity $\mathrm{V}_{\mathrm{Hn}} \approx \mathrm{c}_{\mathrm{HII}} \mathrm{M}_{\mathrm{Hu}} / \mathrm{M}_{\text {neutral }}$ (the "rocket" effect). Photons with a wavelength longer than 912 A, which can penetrate beyond the HII region, are generated in greater abundance than shorter wavelength ionizing photons and can heat cloud surfaces in photo-dissociation regions (PDRs) to about $10^{3} \mathrm{~K}$, producing low-velocity ablation flows and a mild rocket effect.

As shown by Bally and Scoville (1980), clouds can be accelerated to about $5 \mathrm{~km} \mathrm{~s}^{-1}$ if $1 / 4$ of their mass is ablated by ionization, and near the sound speed in ionized gas if $1 / 2$ of the mass is ablated. The dense gas in Orion tends to be seen at higher velocities than the low density gas. If both the Orion A and Orion B clouds are located behind the OB association, then a radial acceleration away from its center produces a positive radial velocity shift for the accelerated gas. This geometry is consistent with the pattern of illumination on the surface of the L1641 region, 
which suggests that it lies behind the illuminating stars. Furthermore, M42 is known to lie in front of its associated cloud. This geometry is also possible for Orion B, although in this case the evidence is less clear.

Ionizing radiation typically converts photon energy into kinetic energy with an efficiency greater than 0.001 (Tielens and Hollenbach 1985). The Lyman continuum luminosity of an 07 star is about $10^{49}$ photons $\mathrm{sec}^{-1}$, so that an estimate of the total present Lyman

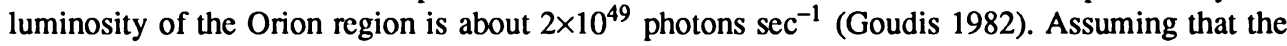
clouds subtend $10 \%$ of the entire sky as seen from the ionizing stars, the kinetic energy injection rate due to ionization is more than $10 \mathrm{~L}_{\odot}$, as much as the value for supemova/stellar wind power injection. Note that the magnitude of both effects depend on inaccurate estimates of small efficiency factors.

OB associations can disrupt giant molecular cloud complexes. Evidence for disruption has been presented by Bally and Scoville (1980) and Leisawitz (1989). Winds, supernovae, and ionization can disperse much of the molecular cloud into smaller accelerated cloud fragments and expanding $\mathrm{HI}$ clouds formed by the recombined gas ablated from the molecular clouds. The cometary shapes of clouds which point away from the center of the OB association and the large-scale velocity gradients indicate that cloud disruption is taking place in the Orion region.

\section{(b) OB Associations and the Evolution of Molecular Clouds}

Within $500 \mathrm{pc}$ of the Sun, there are two other large OB associations besides Orion Per OB2 $(d=350 \mathrm{pc})$ and the Sco-Cen association $(\mathrm{d}=125$ to $200 \mathrm{pc}$; Blaauw 1964; deGeuss 1989). Like Orion, these OB associations are surrounded by massive expanding shells of atomic hydrogen, and are associated with GMC's. All clouds within $700 \mathrm{pc}$ of the Sun having $\mathrm{M}>$ $10^{4} \mathrm{M}_{\odot}$ are associated with $\mathrm{OB}$ associations and large $\mathrm{HI}$ shells. For example, the $\rho$-Oph cloud (associated with the upper-Sco sub-group of the Sco-Cen OB association) exhibits wind-blown and cometary structure with the densest gas and active site of star formation facing the OB stars. The Sco-Cen association is surrounded by a network of nested HI shells which subtend over a steradian as seen from the Sun. We suggest that cloud crushing, ablation, and acceleration produced by $\mathrm{OB}$ associations is responsible for many of the structural and kinematic features of the molecular gas within the Solar neighborhood.

In the Solar neighborhood, the mean separation between GMCs, OB associations, and supershells having ages less than $10^{7}$ years is about $300 \mathrm{pc}$. The associated shells expand with a velocity of order 10 to $40 \mathrm{~km} \mathrm{~s}^{-1}$ into the low-density medium. The distribution of $\mathrm{O}, \mathrm{B}$, and A stars and molecular clouds lying within $800 \mathrm{pc}$ of the Sun appears to define an expanding, tilted system, the Gould Belt, whose center of expansion and age seems to coincide with the 25 Myr old Taurus-Cas group of B and A stars (Blaauw 1964; Olano and Poppel 1987). The nearby HI shells expand with a typical velocity of $10 \mathrm{~km} \mathrm{~s}^{-1}$. If their motion is approximated as a momentum-conserving flow driven by a quasi-steady wind (a reasonable approximation for shells driven by multiple supernovae - McCray and Kafatos, 1987), the shells intersect on a time scale of order $30 \mathrm{Myr}$. These conclusions are only slightly altered by the inclusion of the shear produced by the differential rotation of the Galaxy (Tenorio-Tagle and Palous 1987). Thus no point within the Solar neighborhood remains unaffected by OB-association-driven blast waves for a time longer than this. Supershells must therefore play an important role in the evolution of molecular clouds and the ISM in the Solar neighborhood.

The rocket effect produced by OB stars may be responsible for much of the cloudto-cloud velocity dispersion observed to in the Galaxy. Stark (1979) and Stark and Brand (1988) 
noted that the molecular cloud population is characterized by a $1-\mathrm{d}$ ms cloud-to-cloud velocity dispersion of 6 to $8 \mathrm{~km} \mathrm{~s}^{-1}$. A feature of the velocity dispersion is that it is approximately constant for clouds ranging in mass from under $10^{2} \mathrm{M}_{\odot}$ to over $10^{5} \mathrm{M}_{0}$, implying that the cloud population does not reach collisional equipartition of energy, except for the most massive clouds (Stark 1979). The ISM of the Galaxy contains about $2 \times 10^{9} \mathrm{M}_{6}$ of $\mathrm{HI}$ and a similar amount of $\mathrm{H}_{2}$. Assuming that most of the observed $\mathrm{HI}$ has been ablated from molecular clouds in champagne flows during a molecular cloud dissipation time, the rocket effect should have accelerated the molecular cloud population to a velocity of order the sound speed in ionized gas since the masses of the two phases are similar. This is, in fact, what seems to occur. The total Lyman continuum production rate in our Galaxy is about $Q_{10 t} \approx 5 \times 10^{52}$ photons per second (Smith et al. 1978), each with over $13.6 \mathrm{eV}$ of energy. The total kinetic energy content of GMCs is $1.5 \mathrm{M}_{\mathrm{tot}} \sigma_{1 \mathrm{D}}^{2} \approx 3 \times 10^{54}$ ergs. If on average the conversion efficiency of UV photon energy into kinetic energy is 0.001 , then the Lyman continuum luminosity of the Galaxy can sustain the observed cloud-to-cloud dispersion if the dissipation timescale for random cloud motions is over $10^{8}$ years. Although at any one time only a very small fraction of the ISM is ionized $(<1 \%)$, photo-ionization is the dominant mechanism for converting $\mathrm{H}_{2}$ into $\mathrm{HI}$, with an overall Galactic rate of $\sim 20$ to 100 $\mathrm{M}_{0} \mathrm{yr}^{-1}$. The cloud destruction time scale for the $2 \times 10^{9} \mathrm{M}_{6}$ of $\mathrm{H}_{2}$ in the Galaxy due to interactions with OB stars is of order $10^{7}$ to $10^{8}$ years. In order to maintain the ISM in a quasi-steady state, molecular clouds must reform at a similar rate.

Expanding supcrbubbles may provide an efficient mechanism which sweeps up low density HI from the ISM into dense shells which become gravitationally unstable at a characteristic mass scale of order $10^{5} \mathrm{M}_{\odot}$ (McCray and Kafatos 1987; Tenorio-Tagle and Palous 1987; MacLow, McCray, and Norman 1989). All-sky surveys of the distribution of HI have shown that much of the HI consists of shells or partial shells (the supershells and "worms" of Heiles, 1984). These features of the $21 \mathrm{~cm}$ sky may correspond to the layers of low density gas swept up by the expanding shocks associated with superbubbles surrounding $O B$ associations. McCray and Kafatos (1987) demonstrate that as supershells sweep-up more gas and decelerate, they become unstable to sclf-gravitation. For conditions appropriate to the Solar vicinity, the onset of this instability takes place at a characteristic mass scale of order $5 \times 10^{4} \mathrm{M}_{6}$, essentially the mass of nearby GMCs.

Cooling of the swept-up layers of the expanding supershells should lead to the conversion of $\mathrm{HI}$ into $\mathrm{H}_{2}$. It is thus possible that the youngest molecular clouds may form from gravitational instabilities of old expanding supershells. A prediction of this theory of cloud formation is that the densest parts of old HI shells should contain molecular cores. There is some observational evidence that this is the case - many of the small high latitude molecular clouds discovered by Blitz, Magnani, and Mundy (1984) are located along the HI shells produced by the nearest $\mathrm{OB}$ associations in Sco-Cen, Perseus, and Orion.

Clouds formed by the gravitational fragmentation of old shells may be further compressed by self-gravity or the influence of younger OB groups. The mutual gravitational interaction of the clouds produced by shell fragmentation may be enhanced by swing amplification, streaming motion, and magnetic effects, which are more important in spiral arms. In a spiral arm, the resulting cloud build-up leads to the formation of much more massive cloud complexes than those in the Solar neighborhood. Spiral arm GMCs may produce much more energetic $O B$ associations than those currently near the Sun. Such massive OB groups must have been responsible for the $O B$ association which has set into motion the Gould Belt we see surrounding us. The current generation of $O B$ associations near the Sun may be the result of secondary star formation resulting from the fragmentation of the expanding network of cloud which makes up the Gould Belt. 
Our observations of molecular clouds imply that massive stars play an important role in determining cloud structure and kinematics. Recently-formed stars still embedded inside clouds produce shocks which contribute to cloud support and regulate the cloud collapse rate. On large scales, ionization ablates and accelerates clouds by the rocket effect. The expanding ionization fronts drive shocks into the gas which may trigger gravitational collapse and induced star formation. The stellar winds and supernovae produced by massive stars in OB associations generate large-diameter superbubbles and supershells which sweep up the low density ISM. Cooling and compression in the shells may lead to molecule formation - deceleration and gravitational instability eventually leads to the formation of $10^{5} \mathrm{M}_{6}$ clouds. These GMCs form new $\mathrm{OB}$ associations, continuing the evolutionary cycle. Galactic scale processes such as tidal shear, streaming and orbit crowding in spiral arms, mutual gravitational and magnetic interactions between GMCs and swing amplification modulate this cycle to produce the observed structures in the Galaxy.

\section{REFERENCES}

Bally, J., Langer, W.D, Wilson, R.W. and Stark, A.A. 1987 Ap.J.(Letters), 312, L45.

Bally, J., and Scoville, N.Z. 1980, Ap.J., 239, 121.

Bertoldi, F. 1989a, Ph.D. Thesis, University of California, Berkeley.

Bertoldi, F. 1989b, Ap.J., 346, 735.

Bertoldi, F., and McKee, C.F. 1990, Ap.J., 354, 529.

Blaauw, A. 1964, Ann.Rev.Astr.Ap., 2, 213.

Blitz, L., and Stark, A.A. 1986, Ap.J. (Letters), 300, L89.

Blitz, L., Magnani, L., and Mundy, L. 1984, Ap.J. (Letters), 282, L9.

Bedogni, R., and Woodward, P.R. 1990, Astron. Astrophys., 231, 481.

Bodenheimer, P., Tenorio-Tagle, G., and York, H.W. 1979, Astron. Astrophys., 233, 85.

Cowie, L.L., Songaila, A., and York,D.G. 1979, Ap.J., 230, 469.

Fukui, Y. 1989, in ESO Workshop on Low Mass Star Formation and Pre-Main Sequence Objects, ed: B. Reipurth (ESO Garching).

deGeuss, E. 1988 Ph.D. Thesis, Leiden.

Goudis, C. 1982, The Orion Complex: A Case Study of Interstellar Matter, (Dordrecht: D. Reidel).

Heiles, C. 1984, Ap.J.Suppl., 55, 585.

Lada, E.A. 1989, Ph.D. Thesis, University of Texas, Austin.

Lada, E.A., Bally, J., and Stark, A.A. 1990, Ap.J., (in press).

Leisawitz, D., Bash, F.N., and Thaddeus, P. 1989, Ap.J.Suppl., 70, 731.

MacLow, M., and McCray, R., and Norman, C. 1989, Ap.J., 337, 141.

McCray, R., and Kafatos, M. 1987, Ap.J., 317, 190.

Myers, P.C., and Benson, P.J. 1983, Ap.J., 266, 309.

Olano, C.A., and Poeppel 1987, Astron. Astrophys., 179, 202.

Reipurth, B. 1989, Nature, 340, 42.

Sivan, J.P. 1974, Astron. Astrophys. (Suppl.), 16, 163.

Smith, L.F. ,Biermann, P., and Mezger, P.G. 1978,Astron. Astrophys., 66, 65.

Stark, A.A. 1979, Ph.D. Thesis, Princeton University.

Stark, A.A., and Brand, J. 1989, Ap.J., 339, 763.

Tenorio-Tagle, G., and Palous, J. 1989, Astron. Astrophys., 186, 287.

Tielens, A.G.G.M., and Hollenbach, D.J. 1985, Ap.J., 291, 722.

Ungerechts, H., and Thaddeus, P. 1987, Ap.J.Suppl., 63, 645. 